\title{
Identification of Babbitt Damage and Excessive Clearance in Journal Bearings through an Intelligent Recognition Approach
}

\author{
Joel Pino Gómez ${ }^{1}$, Fidel E. Hernández Montero ${ }^{2}$ \\ Technological University of Havana (Cujae) \\ Havana, Cuba
}

\author{
Julio C. Gómez Mancilla ${ }^{3}$, Yenny Villuendas Rey ${ }^{4}$ \\ Instituto Politécnico Nacional (IPN) \\ CDMX, México
}

\begin{abstract}
Journal bearings play an important role on many rotating machines placed on industrial environments, especially in steam turbines of thermoelectric power plants. Babbitt damage (BD) and excessive clearance (C) are usual faults of steam turbine journal bearings. This paper is focused on achieving an effective identification of these faults through an intelligent recognition approach. The work was carried out through the processing of real data obtained from an industrial environment. In this work, a feature selection procedure was applied in order to choose the features more suitable to identify the faults. This feature selection procedure was performed through the computation of typical testors, which allows working with both quantitative and qualitative features. The classification tasks were carried out by using Nearest Neighbors, Voting Algorithm, Naïve Associative Classifier and Assisted Classification for Imbalance Data techniques. Several performance measures were computed and used in order to assess the classification effectiveness. The achieved results (e.g., six performance measures were above 0.998 ) showed the convenience of applying pattern recognition techniques to the automatic identification of $\mathrm{BD}$ and $\mathrm{C}$.
\end{abstract}

Keywords-Journal bearing; Babbitt damage; excessive clearance; fault identification; feature selection; supervised classification

\section{INTRODUCTION}

Many rotating machine failures are related to bearing faults [1-5]. Journal bearings (JB) are usually found in heavy industries that include large rotating machines, whose early fault identification can yield a favorable impact on plant availability [6-9]. The stable operation of JB requires the clearance not to exceed the operating boundaries. If the clearance is out of bounds, mechanical instabilities in the shaft rotation, such as oil whirl, oil whip, looseness, or journal-tobearing contact can arise $[10,11]$. Mechanical stress due to these instabilities can also cause damage on the bearing babbitt surface, particularity, the oil whip is an unsafe operation that may cause severe damage on the machine [9-11]. Journal bearings are inspected during the maintenance process by a clearance measuring procedure, as well as an accurate examination of the babbitt surface; if a high-level damage occurs then a re-babbitting procedure will be necessary. Babbitt damage (BD) and excessive clearance (C) have been widely addressed in several researches [9-13].
Several classification methods have been applied on the automatic diagnosis of JB faults [14-33]. However, no research work addressing the automatic detection of $\mathrm{BD}$ and $\mathrm{C}$ in cylindrical journal bearings, through data gathered from real industrial environments, have been found by the authors of this paper, despite cylindrical journal bearings (CJB) are among the most common types of hydrodynamic journal bearings used by the turbomachinery $[34,35]$.

Even though the staff specialized in the diagnostic of JB faults evaluates a wide range of features expressed by numerical, ordinal and nominal variables (mixed features) [3638], most research works addressing JB faults use only numerical variables, which are mainly vibration features extracted from both time and frequency domains [14-33]. Expert knowledge was previously considered in [38], but such a work only addressed the feature selection task.

This paper is focused on the automatic identification of $\mathrm{BD}$ and $\mathrm{C}$ in CJB by means of the processing of mixed features extracted from data gathered at a real industrial environment. The proposed methodology involves a feature selection procedure as a primary step, and then, the application of several classifiers. The mixed features processing is provided by the application of the Logical Combinatorial Pattern Recognition approach (LCPR) [39].

This paper is organized as follows: Section II provides a brief summary of previous works on the automatic fault diagnosis of JB. Section III presents some concepts and tools of the LCPR approach. The main features of the JB vibration spectrum that are traditionally used for diagnostics purposes are presented in Section IV. Section V presents the features and datasets used in this work. Section VI describes the proposed methodology. Section VII shows the main results and related discussions, and Section VIII presents the conclusions and future works.

\section{REVIEW OF PREVIOUS WORKS ON AUTOMATIC FAULT DIAGNOSIS OF JOURNAL BEARINGS}

Both supervised and unsupervised classification methods have been applied on the automatic diagnosis of JB faults [1433]. While most of such works have used either data collected from a testbench [16-31] or data obtained from numerical models of faults [32, 33], just a few has used data gathered from a real life environment [14, 15]. For example, in [32, 33],

*Corresponding Author 
different conditions of ovalization and wear were simulated by using numerical models. The classification methods used in these works were based on Convolutional Neural Networks (CNN) and resulted in a good accuracy. In [16-18], several faults (related to oil supply, looseness and bearing surface damages), induced in a journal bearing testbench, were diagnosed by means of Artificial Neural Networks (ANN) and Deep Neural Networks (DNN); a high effectiveness was achieved. Different friction and wear conditions were diagnosed in [24-26]. In these works, two test rigs were used and both Random Forest Classifier (RFC) and Support Vector Machines (SVM) were successfully applied. In [19-23], the effective diagnostic of unbalance, misalignment, rubbing and oil whirl was performed by applying Fisher Discriminant Analysis (FDA), Multilayer Perceptron (MLP), CNN and SVM. The data was gathered from a Bently-Nevada RK4 rotor kit and a feature selection procedure was implemented through the application of the Fisher Discriminant Ratio (FDR), Deep Belief Network (DBN), Kullback-Leibler Divergence (KLD) and Probability of Separation (PoS). In [27], a CNN classifier was applied on contact rubbing, block looseness, rotor unbalance and misalignment diagnostics. The results were compared with those obtained by SVM and a Probabilistic Neural Network (PNN). In that work, two testbenches were used. In [28], Genetic algorithms (GA) and ANN were used in order to identify three different lubrication conditions induced in a journal bearing test bed. The inadequate lubrication, oil starvation, corrosion, metal-to-metal contact, and extreme wear in the main journal bearing of an internal combustion engine were the faults addressed in [29-31]. In these works, k-Nearest Neighbor (kNN), Fisher Linear Discriminant (FLD), ANN and SVN classifiers were satisfactory applied. In [15], DBN, MLP, FDA and Self-Organizing Map (SOM) were the techniques used in order to diagnose the misalignment, the rubbing and the oil whirl produced in both a Bently-Nevada RK4 rotor kit and the journal bearings of a $500 \mathrm{MW}$ steam turbine in a power plant. Several malfunctions like friction, abnormal lubrication and $\mathrm{C}$ were accurately diagnosed by means of Linear Discriminant Analysis (LDA), FDA and SVM techniques [14]. Such work was performed on induction motors and generators under full load conditions.

However, none of the previously mentioned paper address the automatic detection of $\mathrm{BD}$ and $\mathrm{C}$ in $\mathrm{CJB}$, through data gathered from real industrial environments.

\section{BRIEF INTRODUCTION TO LCPR APPROACH}

LCPR constitutes an approach suitable to deal with mixed data (i.e., both quantitative and qualitative features) in feature selection and pattern classification applications. This approach provides several useful tools for processing mixed and incomplete data [39]. LCPR involves multiples comparison criteria to establish comparisons between the values of a feature. A comparison criterion (CC) is a mathematical formulation that allows for computing the similarity or dissimilarity between the values taken by a feature for two different objects. The following $\mathrm{CCs}$ are two examples that allow for determining the dissimilarity between either nominal or numerical features, respectively:

$$
\begin{aligned}
& C C_{1}(X s(O i), X s(O j))= \begin{cases}1 & \text { if } X s(O i) \neq X s(O j) \\
0 & \text { otherwise }\end{cases} \\
& C C_{2}(X s(O i), X s(O j))= \begin{cases}1 & \text { if }|X s(O i)-X s(O j)|>\sigma_{s} \\
0 & \text { otherwise }\end{cases}
\end{aligned}
$$

where $X s(O i)$ and $X s(O j)$ are the values of the feature $s$ for the objects $O i$ and $O j$, respectively, and $\sigma_{s}$ is the standard deviation of the values taken by the feature $s$, in case of being a numerical feature. The output takes values ' 0 ' or ' 1 ' indicating that the comparison results are similar or dissimilar, respectively. For example, Table 1 presents three objects described by two features: the feature 1 (a nominal feature) and the feature 2 (a numerical feature).

Then, $\mathrm{CC} 1$ can be used in order to compare the values taken by feature 1 and $C C_{2}$ can be used for the values taken by feature 2. Assuming that the standard deviation of feature 2 is $\sigma_{2}=0.28$, the comparison $(\mathrm{Cr})$ between the three objects results yields:

$$
C r=\left[\begin{array}{ll}
1 & 1 \\
0 & 1 \\
1 & 0
\end{array}\right]
$$

where the row 1 is the result of comparing $\mathrm{O}_{1}$ and $\mathrm{O}_{2}$ : "dissimilar" for both features. The row 2 is the result of comparing $\mathrm{O}_{1}$ and $\mathrm{O}_{3}$ : "similar" for feature 1 and "dissimilar" for feature 2. And the row 3 is the result of comparing $\mathrm{O} 2$ and O3: "dissimilar" for feature 1 and "similar" for feature 2.

The feature selection can be accomplished by means of a useful tool: The Typical Testor (TT) computation. A testor (T) is defined as a subset of features that allows for differentiating between any two objects that belong to different classes; CCs defined for the comparison of such features are used. A TT is an irreducible T; that is, if any feature of a TT is removed, then the TT stops being a T [39]. Therefore, a TT is the most compact form in which a testor can appear. In a pattern recognition problem, the set of all TTs contains all the minimum-length subsets of features that allow for class differentiation. The TTs make contribution to both the classification process and the selection of only the more significant features.

Sometimes, the computation of the whole set of TTs can take long times. This is due to the algorithms need to check several features subsets, bounded by the exponential of the number of features. Undoubtedly, this is a non-polynomial problem that could incur in a high computational cost. Several algorithms or methods have been developed for the minimization the TT searching time [40]. In this work, the TTs were computed by means of one of the most powerful algorithms: the fast-BR algorithm [41].

TABLE I. EXAMPLE OF OBJECT DESCRIPTION

\begin{tabular}{|l|l|l|}
\hline Object & Feature 1 & Feature 2 \\
\hline $\mathrm{O}_{1}$ & $1 \mathrm{Xh}$ & 3.07 \\
\hline $\mathrm{O}_{2}$ & $2 \mathrm{Xv}$ & 1.78 \\
\hline $\mathrm{O}_{3}$ & $1 \mathrm{Xh}$ & 2.03 \\
\hline
\end{tabular}


According to the pattern classification task, testors bring out an idea about which features are more significant or which features provide more information. Accordingly, the importance of a feature can be assessed by the number of TTs that include such a feature [39]. That is,

$$
P(x)=\frac{\omega(x)}{\omega}
$$

where $\omega$ is the number of TTs and $\omega(x)$ is the number of TTs that include the feature $x$. According to equation (3), the higher the number of TTs that include a feature, the higher the importance of such a feature. In addition, the feature importance can be assessed through the dimensions of the TTs that include such a feature [39]. That is,

$$
L(x)=\frac{\sum_{t \in \psi(x)} \frac{1}{t}}{\psi(x)}
$$

where $t$ is the number of features of each TT including the feature $x$, and $\psi(x)$ is the number of TTs that include the feature $x$. According to equation (4), a feature is more important as it is found in shorter TTs. Finally, the feature importance can be expressed as follows [39]:

$\rho(x)=\alpha P(x)+\beta L(x)$

where $\alpha$ and $\beta$ are weighting coefficients of $P(x)$ and $L(x)$, respectively. Then, the features selection is completed by removing the resulting features with importance values below thresholds empirically established.

\section{FEATURES OF THE JOURNAL BEARING VIBRATION SPECTRUM}

Vibration analysis is essential for the condition evaluation of journal bearings [37]. Frequency domain representations of vibration signals bring out features very significant to JB fault diagnosis [21]. The spectrum of the journal bearing vibration signals usually exhibits several harmonics. If $X$ is the value of the rotational speed in $\mathrm{Hz}$, then some features of the vibration spectrum that are usually inspected for diagnostics purposes are [21, 37]: the synchronous spectral component (the amplitude of the spectral component at frequency $1 X$ ), its harmonics (the amplitudes of the spectral components at frequencies corresponding to integer multiples of $1 X$, e.g., $2 X$, $3 X, \ldots$ ), its inter-harmonics (the amplitudes of the spectral components between successive harmonics, e.g., 1.5X), and its sub-synchronous (the amplitudes of the spectral components under frequency $1 X$, e.g., $0.4 X$ ).

Figure 1 shows an example of a real JB velocity vibration spectrum, where some of the aforementioned features can be seen. Taking into account that the rotational speed is 3600 revolutions per minute $(60 \mathrm{~Hz})$, the more predominant spectral components are the harmonics $1 X, 2 X, 3 X, 4 X, 6 X$ and $8 X$. These features could be used for automatic JB fault diagnosis $[20,21,33]$; however, in several cases many of them could either appear at very low amplitudes or be not visible in the spectrum (e.g., the sub-synchronous, inter-harmonics or some harmonics components do not appear in the spectrum shown in
Figure 1). In such cases, these features will not be contributing with meaningful information to the diagnosis process.

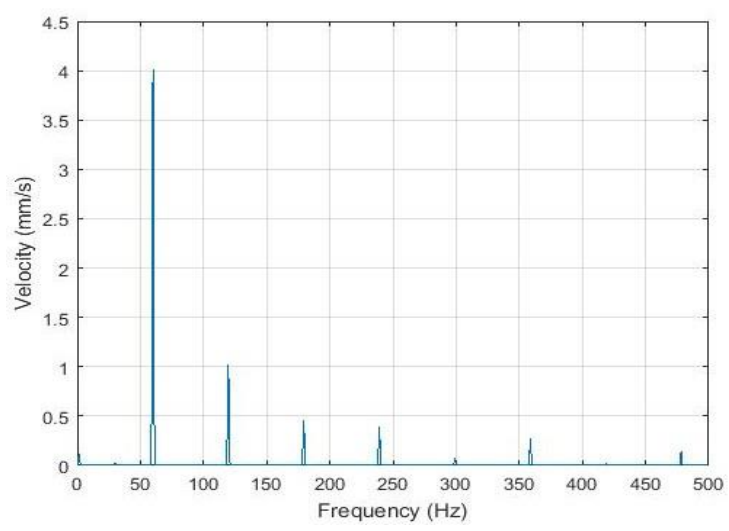

Fig. 1. Velocity Vibration Spectrum of a Real JB.

Other features, such as ratios between the aforementioned features, as well as different statistical measures, can also be extracted from the vibration spectrum [20,31].

\section{FEATURES AND DATASETS SUPPORTING THE CLASSIFICATION TASKS}

From the set of features of the velocity vibration spectrum, presented in Section 3, this work addressed the use of the features that provided the information more useful to the diagnosis procedure. In this work, a new approach of feature arrangement was applied: while a small number of features is well defined, another group of features is undetermined and will be defined by the spectral components reaching the largest amplitudes. In this work, 35 mixed features were used for the pattern classification task; a brief description of them is presented in Table 2.

The feature 1 was the synchronous spectral component of the horizontal vibration; features from 2 to 6 were the highestamplitude spectral components of the horizontal vibration, regardless the frequencies at which they were given rise; feature 7 was the synchronous spectral component of the vertical vibration; features from 8 to 12 were the highestamplitude spectral components of the vertical vibration, regardless the frequencies at which they were given rise; features from 13 to 17 were the ratios of the highest-amplitude spectral components of the horizontal vibration (features from 2 to 6 ) to the synchronous spectral component of the horizontal vibration (feature 1); features from 18 to 22 were the ratios of the highest-amplitude spectral components of the vertical vibration (features from 8 to 12) to the synchronous spectral component of the vertical vibration (feature 7); feature 23 was the ratio of the synchronous spectral component of the horizontal vibration (feature 1) to the synchronous spectral component of the vertical vibration (feature 7); and features from 24 to 35 were the names (nominal features) of the spectral components denoted by features from 1 to 12 arranged in descendent order according to their values. The nominal features allow for the identification of the spectral components selected as the first 12 features and supply information about their amplitude order. Two examples of the set of features can be found in Table 3 . 
TABLE II. FEATURE DESCRIPTION

\begin{tabular}{|c|c|c|c|}
\hline No. & $\begin{array}{l}\text { Value description } \\
\text { (H: Horizontal Vibration; V: Vertical } \\
\text { Vibration) }\end{array}$ & Frequency & $\begin{array}{l}\text { Domain } \\
\text { (R: Real; N: } \\
\text { Nominal) }\end{array}$ \\
\hline 1 & $\begin{array}{l}\text { amplitude of the synchronous component } \\
\text { (H), } 1 \mathrm{Xh}\end{array}$ & Rotational & $\mathrm{R}$ \\
\hline 2 & $\begin{array}{l}\text { highest amplitude of a spectral component } \\
\text { (H) different to } 1 \mathrm{Xh}\end{array}$ & $?$ & $\mathrm{R}$ \\
\hline 3 & $\begin{array}{l}\text { second highest amplitude of a spectral } \\
\text { component }(\mathrm{H}) \text { different to } 1 \mathrm{Xh}\end{array}$ & $?$ & $\mathrm{R}$ \\
\hline$\vdots$ & $\vdots$ & $\vdots$ & $\vdots$ \\
\hline 6 & $\begin{array}{l}\text { fifth highest amplitude of a spectral } \\
\text { component }(\mathrm{H}) \text { different to } 1 \mathrm{Xh}\end{array}$ & $?$ & $\mathrm{R}$ \\
\hline 7 & $\begin{array}{l}\text { amplitude of the synchronous component } \\
\text { (V), } 1 \mathrm{Xv}\end{array}$ & Rotational & $\mathrm{R}$ \\
\hline 8 & $\begin{array}{l}\text { highest amplitude of a spectral component } \\
\text { (V) different to } 1 \mathrm{XV}\end{array}$ & $?$ & $\mathrm{R}$ \\
\hline 9 & $\begin{array}{l}\text { second highest amplitude of a spectral } \\
\text { component (V) different to } 1 \mathrm{Xv}\end{array}$ & $?$ & $\mathrm{R}$ \\
\hline$\vdots$ & $\vdots$ & $\vdots$ & $\vdots$ \\
\hline 12 & $\begin{array}{l}\text { fifth highest amplitude of a spectral } \\
\text { component }(\mathrm{V}) \text { different to } 1 \mathrm{Xv}\end{array}$ & $?$ & $\mathrm{R}$ \\
\hline 13 & $\begin{array}{l}\text { rate of value of feature } 2 \text { to the value of } \\
\text { feature } 1\end{array}$ & -- & $\mathrm{R}$ \\
\hline 14 & $\begin{array}{l}\text { rate of value of feature } 3 \text { to the value of } \\
\text { feature } 1\end{array}$ & -- & $\mathrm{R}$ \\
\hline$\vdots$ & $\vdots$ & $\vdots$ & $\vdots$ \\
\hline 17 & $\begin{array}{l}\text { rate of value of feature } 6 \text { to the value of } \\
\text { feature } 1\end{array}$ & -- & $\mathrm{R}$ \\
\hline 18 & $\begin{array}{l}\text { rate of value of feature } 8 \text { to the value of } \\
\text { feature } 7\end{array}$ & -- & $\mathrm{R}$ \\
\hline 19 & $\begin{array}{l}\text { rate of value of feature } 9 \text { to the value of } \\
\text { feature } 7\end{array}$ & -- & $\mathrm{R}$ \\
\hline$\vdots$ & $\vdots$ & $\vdots$ & $\vdots$ \\
\hline 22 & $\begin{array}{l}\text { rate of value of feature } 12 \text { to the value of } \\
\text { feature } 7\end{array}$ & -- & $\mathrm{R}$ \\
\hline 23 & $\begin{array}{l}\text { rate of value of feature } 1 \text { to the value of } \\
\text { feature } 7\end{array}$ & -- & $\mathrm{R}$ \\
\hline 24 & $\begin{array}{l}\text { name of the feature from } 1 \text { to } 12 \text { with the } \\
\text { highest amplitude }\end{array}$ & ? & $\mathrm{N}$ \\
\hline 25 & $\begin{array}{l}\text { name of the feature from } 1 \text { to } 12 \text { with the } \\
\text { second highest amplitude }\end{array}$ & $?$ & $\mathrm{~N}$ \\
\hline$\vdots$ & $\vdots$ & $\vdots$ & $\vdots$ \\
\hline 35 & $\begin{array}{l}\text { name of the feature from } 1 \text { to } 12 \text { with the } \\
\text { twelfth highest amplitude }\end{array}$ & $?$ & $\mathrm{~N}$ \\
\hline
\end{tabular}

TABLE III. Two EXAMPLES OF SET OF FEATURES

\begin{tabular}{|l|l|}
\hline Objects & Features Values \\
\hline \multirow{3}{*}{ object 1 1} & $5.03,1.61,1.04,1.04,0.92,0.76,1.43,1.84,1.45,1.24,1.15,0.78$, \\
& $0.32,0.21,0.21,0.18,0.15,1.29,1.02,0.87,0.81,0.55,3.52,1 \mathrm{Xh}$, \\
& $3 \mathrm{Xv}, 2 \mathrm{Xh}, 2 \mathrm{Xv}, 1 \mathrm{Xv}, 4 \mathrm{Xv}, 5 \mathrm{Xv}, 6 \mathrm{Xh}, 3 \mathrm{Xh}, 4 \mathrm{Xh}, 6 \mathrm{Xv}$ \\
\hline \multirow{3}{*}{ object 2 } & $3.13,0.51,0.39,0.32,0.16,0.12,3.27,1.71,1.52,1.27,0.53,0.53$, \\
& $0.16,0.13,0.1,0.05,0.04,0.52,0.46,0.39,0.16,0.16,0.96,1 \mathrm{Xv}$, \\
& $1 \mathrm{Xh}, 2 \mathrm{Xv}, 4 \mathrm{Xv}, 3 \mathrm{Xv}, 6 \mathrm{Xv}, 5 \mathrm{Xv}, 6 \mathrm{Xh}, 2 \mathrm{Xh}, 3 \mathrm{Xh}, 5 \mathrm{Xh}, 4 \mathrm{Xh}$ \\
\hline
\end{tabular}

The dataset supporting this research were taken from diagnostics and maintenance reports of a $100 \mathrm{MW}$ steam turbine running for three years in an active thermoelectric power plant. We cannot disclose the name of the thermoelectric plant due to confidentiality issues. Data was collected from four journal bearings that were affected by five different fault conditions: $\mathrm{C}, \mathrm{BD}$ in the bottom half of the journal bearing (B), BD in the top half of the journal bearing (T), faults B and C occurring simultaneously (BC), and faults $\mathrm{B}, \mathrm{C}$ and $\mathrm{T}$ occurring simultaneously (BCT). Data corresponding to healthy condition of operation was not available. The total number of measurements (objects) to work with was 3314 .

Each object description is expressed by the set of 35 features proposed and described in previous section. These features were extracted from the JB absolute vibration measurements taken at both horizontal and vertical directions, at a sampling frequency equal to $1 \mathrm{kHz}$. Such measurements were performed and stored by means of the online monitoring system installed at the thermoelectric power plant. The online monitoring system and the velocity sensor installed for the vibration acquisition were the VIBROCONTROL 4000 and BK VIBRO VS-079, respectively. Each measurement consisted of an 800-lines spectrum.

The object distribution is shown in Table 4. This table reveals that the object quantities were imbalanced for the five classes of faults, with an Imbalance Ratio IR=6.61.

TABLE IV. OBJECT DISTRIBUTION

\begin{tabular}{|l|l|}
\hline Fault Conditions & Objects Numbers \\
\hline B & 431 \\
\hline C & 708 \\
\hline T & 213 \\
\hline BC & 1409 \\
\hline BCT & 553 \\
\hline
\end{tabular}

\section{MethodolOGY}

The aim of this research is to identify automatically the BD and $\mathrm{C}$ in $\mathrm{CJB}$ through data taken from real industrial environments. Figure 2 shows a scheme of the methodology developed in order to accomplish this goal; this methodology is described as follows.

Firstly, a feature selection process addressing the determination of the TTs from the set of 35 features presented in the section 4, was implemented. The algorithm used in order to search the TTs was the fast-BR [41]. The feature importance was computed by applying the equation (5) for both parameters $\alpha$ and $\beta$ being equal to 0.5 . After the implementation of several tests, the threshold for feature selection was chosen to be the difference between the mean and the half of the standard deviation of the computed importance records.

Afterwards, a classification strategy was implemented: on one hand, the classification procedure was carried out by using only the important (selected) features and, on the other hand, the classification procedure was performed by using the whole set features. This strategy will reveal how effective the implemented feature selection procedure was. 


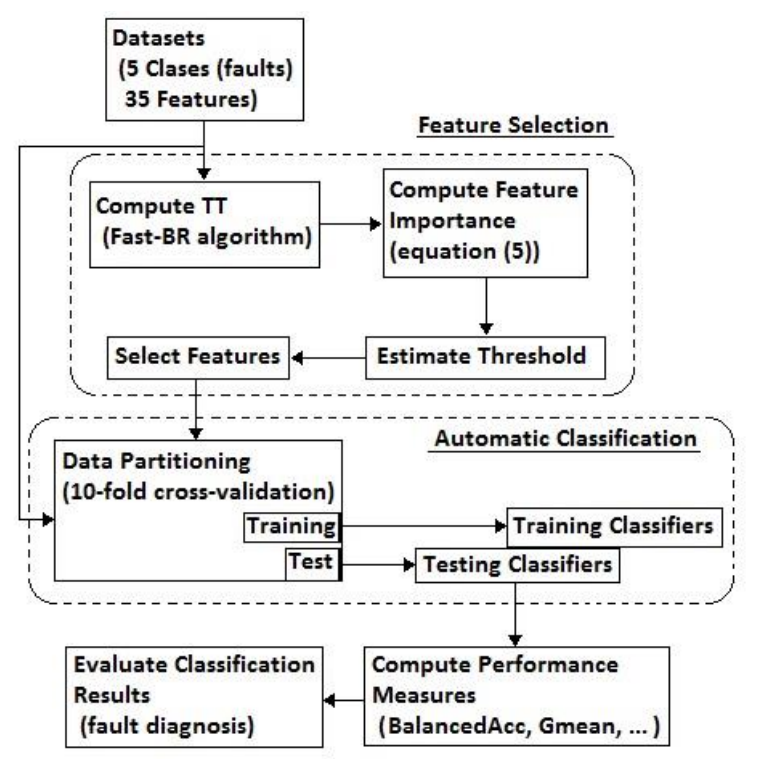

Fig. 2. Methodology.

Four classification techniques were applied: kNN [42], Voting Algorithm (ALVOT) [39], Naïve Associative Classifier (NAC) [43] and Assisted Classification for Imbalance Data (ACID) [44]. These methods are very suitable to be applied when mixed-data processing is required and they have exhibited high effectiveness in different scenarios. The initialization parameters and dissimilarity function of the ALVOT and NAC algorithms were the same as those presented in [43]. For the $\mathrm{kNN}$ and ACID classifiers the dissimilarity function used was HEOM [45]. Table 5 summarizes the parameters used for the compared classifiers.

The classification tests were carried out on the Experimental Platform for Intelligent Computing (EPIC) [46]. Although this platform is not among the most popular tools for intelligent computing experiments (such as WEKA [47] and KEEL [48]), it allows for processing mixed and incomplete data and it provides the classifiers proposed to be applied on this research.

Cross-validation methodology, specifically, the k-fold cross-validation procedure with $\mathrm{k}$ equal to 10 , was applied in order to warrant the reliability of the results. This procedure has been widely employed in the context of pattern recognition, machine learning and data mining, and the most common scheme has been the 10-fold cross-validation [49]. Although the available dataset is imbalanced, the class with the lowest number of objects admits 10 -fold cross-validation.

The performance measures applied for the evaluation of the classification results, given the imbalance of the dataset presented in Table 2, were: the balanced accuracy (BalancedAcc) [50], the geometric mean of the recall measure (Gmean) [51], the macro precision (PrecisionM) [52], the macro geometric mean of the precision and recall measures (GmeasureM) [53] , the macro F-measure (FScoreM) [52] and the kappa (Kappa) statistic [54]. These indexes are good measures of the classifiers' performance and they are recommended to be used in multiclass and imbalanced problems [54].
TABLE V. Classifier PARAMETERS

\begin{tabular}{|l|l|}
\hline Classifier & Parameter values \\
\hline ACID & $N_{p}=25, i t=100, \varepsilon=0.1$, Dissimilarity: HEOM \\
\hline \multirow{4}{*}{ ALVOT } & $\begin{array}{l}\text { SSS: Typical testors, Decision rule: class with maximum } \Gamma_{j}(o), \\
\text { Similarity: } 1 / \mathrm{HEOM} \\
\end{array}$ \\
$\begin{array}{l}\Gamma_{j}(o)=\frac{\sum_{\Omega_{i} \in S} \Gamma_{\Omega_{i}}^{j}(o, y)}{|S|}, \Gamma_{\Omega_{i}}^{j}(o)=\frac{\sum_{y \in T_{j}} \Gamma_{\Omega_{i}}(o, y)}{\left|K_{j}\right|}, \Gamma_{\Omega_{i}}(o, y)=\rho_{y} * \rho_{y} * \\
\beta\left(o, y, \Omega_{i}\right)\end{array}$ \\
\hline $\mathrm{kNN}$ & $k=1$, Dissimilarity: HEOM \\
\hline NAC & $w_{j}=1$ for all features \\
\hline
\end{tabular}

The comparison criteria given by equations (1) and (2) were used in order to compare both nominal and numerical features, respectively, during the feature selection and classification procedures.

\section{RESULTS AND DISCUSSION}

The number of TTs obtained from the set of 35 features presented in the section 4 was 31218 . The feature importance results are shown in Figure 3. In this figure, selected and removed features are shown through colors green and red, respectively. The decision threshold used in order to select the features is the red horizontal line shown in Figure 3. The number of selected and removed features was $23(65.71 \%)$ and $12(34.29 \%)$, respectively. There were no features with importance values equal to zero (each feature was found at least in one TT). These 23 selected features formed a set of features used in order to perform one of the two implemented fault classification procedures. Another fault classification procedure was carried out by using the set of features given by all of features.

The results obtained by the application of the four selected classifiers on the identification of the faults are shown in Figures 4 to 7 (gray color for the results obtained when the whole set of features was used; green color for the results obtained when the selected features were used).

The results obtained from the application of ALVOT classifier are shown in Figure 4. In general, the best performance was obtained when the set of selected features was used, except for the PrecisionM measure, which reached a value slightly higher for the set of all features. With regard to the six performance measures, the application of the ALVOT classifier yielded values higher than $82 \%$ and according to the BalancedAcc and Gmean measures the values were higher than $90 \%$.

The results obtained by means of the NAC classifier are shown in Figure 5. According to the six performance measures, the best results were obtained when the set of selected features was used. The six performance measures show values higher than $92 \%$, which proves that NAC is a good classifier for automatic identification of $\mathrm{BD}$ and $\mathrm{C}$ in CJB.

Figure 6 shows the results obtained by means of the kNN classifier. In this case, the best performance was obtained when the set of all features was used. kNN was an effective classifier for the automatic fault identification, since the six performance measures yielded values higher than $95 \%$. 


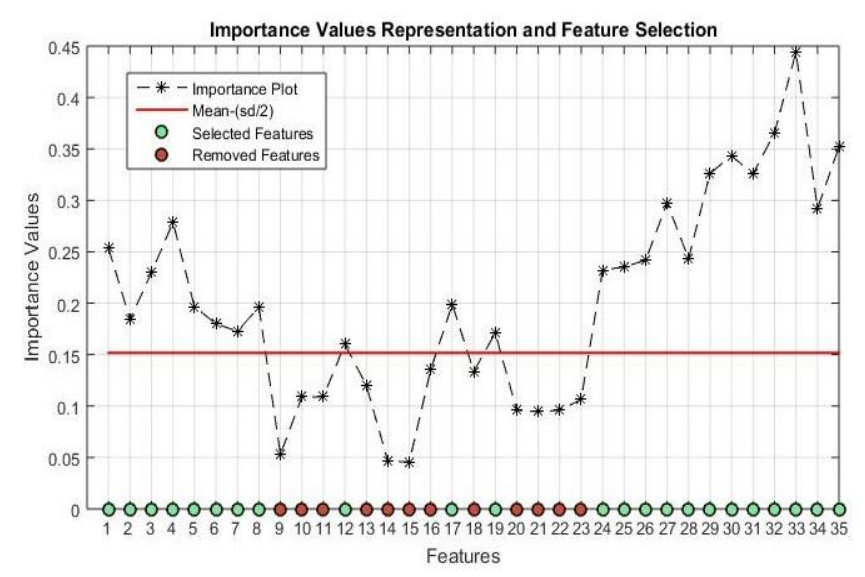

Fig. 3. Feature Importance for the Identification of B, C, T, BC and BCT Faults.

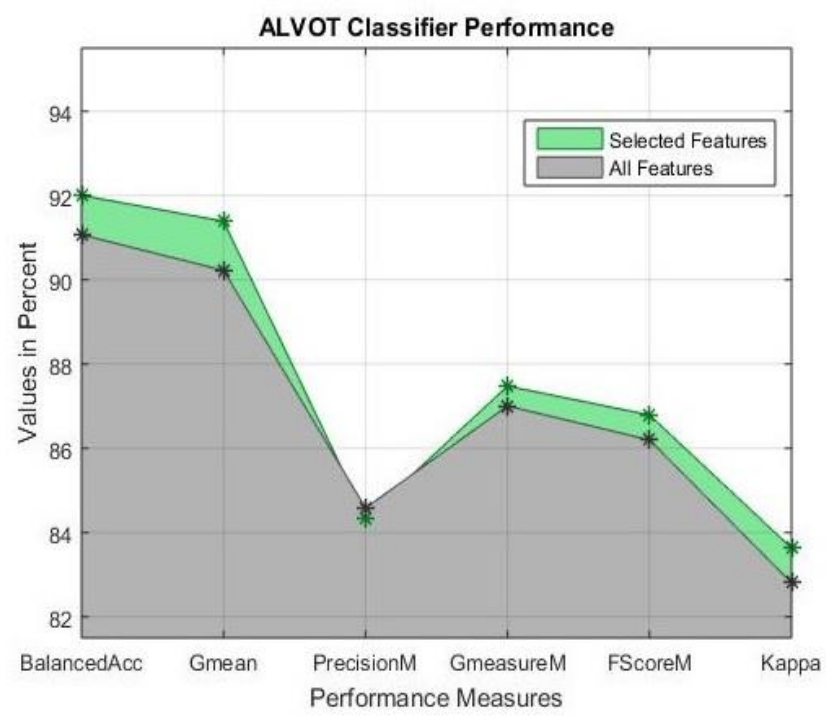

Fig. 4. Results obtained by ALVOT Classifier.

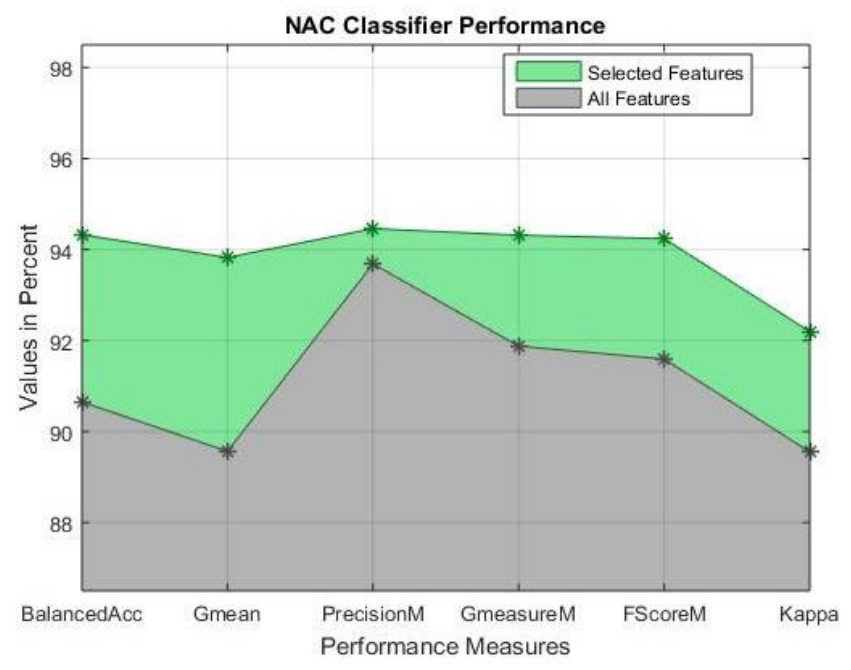

Fig. 5. Results obtained by NAC Classifier.

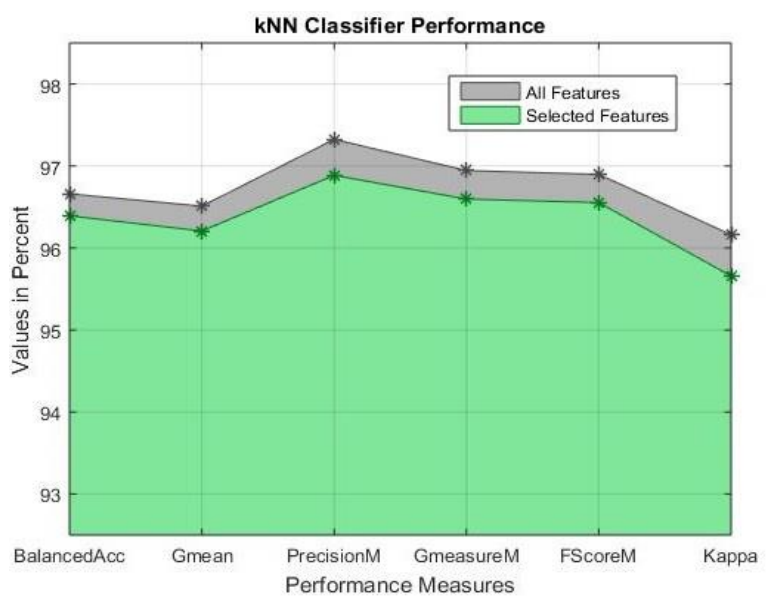

Fig. 6. Results obtained by kNN Classifier.

Figure 7 shows the results obtained when the ACID classifier was applied. In this case, as the six performance measures reveal, the best results were obtained when the set of selected features was used; every computed measure yielded values higher than $99.8 \%$. This classifier also proved to be suitable for the automatic fault identification in CJB.

Summarizing, the values achieved by the computed performance measures proved that the four classifiers are suitable to be applied on fault diagnostics of CJB when the features proposed in this paper are used. It should be noticed that the kNN and ACID classifiers yielded the best performances.

These results validate the methodology proposed for the automatic identification of $\mathrm{BD}$ and $\mathrm{C}$ in $\mathrm{CJB}$. This methodology involves the use of TTs for feature selection and the classifiers ALVOT, NAC, kNN and ACID for fault diagnosis.

These results are highly relevant since they constitute a first report on the automatic identification of $\mathrm{BD}$ and $\mathrm{C}$ in $\mathrm{CJB}$ through data collected at a real industrial environment. Besides, the importance of using mixed features for such purpose was also validated for first time.

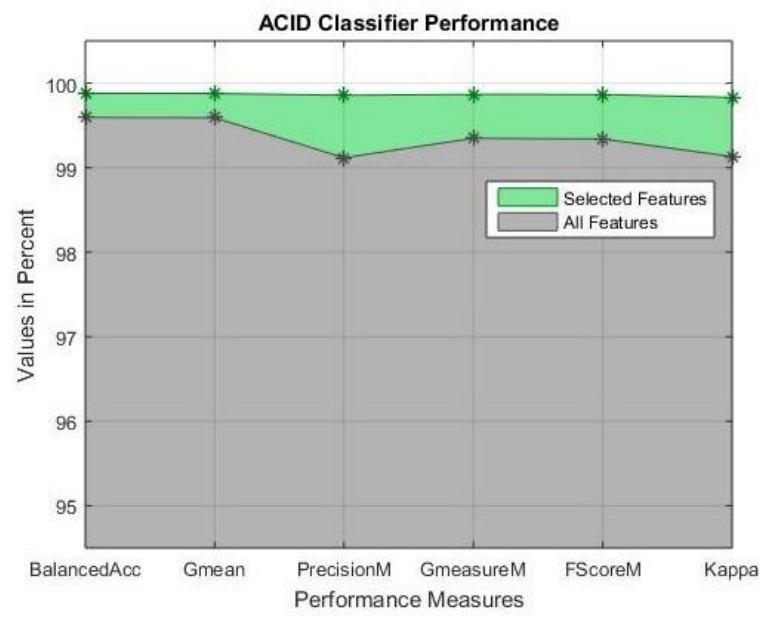

Fig. 7. Results obtained by ACID Classifier. 


\section{CONCLUSIONS}

This paper presents the results of a study about the identification of $\mathrm{BD}$ and $\mathrm{C}$ in journal bearing through the use of features extracted from the vibration spectrum. In this work, the faults and data processed have come from four journal bearings of a 100 MW steam turbine placed in an active thermoelectric power plant.

To the best of our knowledge, this work constitutes the first study addressing the automatic classification of $\mathrm{BD}$ and $\mathrm{C}$ in CJB placed in a real industrial environment. Besides, the use of only the more useful features that could be extracted from the vibration spectrum, as well as the use of both numerical and nominal features (all representing the expert's knowledge) for JB fault identification is proposed as a new methodological approach that led to remarkable results.

The classification process, which consisted in using four different classifiers and working with both selected features and the whole set of features, was very successful, since the effectiveness obtained was very high. In particular, the highest performance (99.8\%) was achieved by ACID algorithm. This algorithm and $\mathrm{kNN}$ are the classifiers recommended to be used for the identification of $\mathrm{BD}$ and $\mathrm{C}$ in journals bearings. The search of typical testors is recommended for performing feature selection.

Several significant novelties were presented in this paper: the use of real-world dataset for CJB fault identification; the use of a new set of features, involving both numerical and nominal features for fault identification; the implementation of a feature selection procedure for improving the classification tasks; and the application of two effective classifiers (NAC and ACID) on the automatic fault diagnosis of machinery.

As future work we want to address other feature selection techniques, as well as other strategies for computing feature importance.

\section{ACKNOWLEDGMENT}

The authors would like to thank the support given by the EPIC research team, from IPN, Mexico, as well as the contributions of the diagnostic and maintenance experts: Julio González Martínez, Yuritza Cruz Guzmán, Jorge C. Arce Miranda and María Antonia Téllez. The authors thank the management of the thermoelectric power plant for providing access to the data used in accordance with the confidentiality agreement signed. The authors also thank the Instituto Politécnico Nacional (Secretaría Académica, COFAA, SIP, ESIME and CIDETEC), the CONACyT, and SNI for their economical support to develop this work.

\section{REFERENCES}

[1] L. Ruonan, Y. Boyuan, Z. Enrico, and C. Xuefeng, "Artificial intelligence for fault diagnosis of rotating machinery: A review," Mechanical Systems and Signal Processing, vol. 108, pp. 33-47, 2018.

[2] G. Królczyk, Z. Li, and J. A. Antonino Daviu, "Fault Diagnosis of Rotating Machine," Applied Sciences, vol. 10, no. 6, p. 4, 2020.

[3] T. Qiang, P. Flores, and H. M. Lankarani, "A comprehensive survey of the analytical, numerical and experimental methodologies for dynamics systems with clearance or imperfect joints," Mechanism and Machine Theory vol. 122, pp. 1-57, 2018.
[4] S. Schmidt, P. S. Heyns, and K. C. Gryllias, "A pre-processing methodology to enhance novel information for rotating machine diagnostics," Mechanical Systems and Signal Processing, vol. 124, pp. 541-561, 2019.

[5] Y. Wei, Y. Li, M. Xu, and W. Huang, "A review of early fault diagnosis approaches and their applications in rotating machinery," Entropy, vol. 21, no. 4, p. 26, 2019.

[6] J. Pino Gómez et al., "Maintenance importance of mechanical elements and faults in steam turbines. Data history analysis," Ingeniería Energética, vol. 38, no. 2, pp. 106-114, 2017.

[7] N. Ding, H. Li, Z. Yin, and F. Jiang, "A novel method for journal bearing degradation evaluation and remaining useful life prediction under different working conditions," Measurement, vol. 177, 2021.

[8] H. Al-Mosawy, H. Jamali, and M. Tolephih, "Effects of linear modification on the performance of finite length journal bearings," in IOP Conference Series: Materials Science and Engineering, 2021.

[9] L. Zhang, H. Xu, S. Zhang, and S. Pei, "A radial clearance adjustable bearing reduces the vibration response of the rotor system during acceleration," Tribology International, vol. 144, p. 15, 2020.

[10] J. Junyeong et al., "Monitoring Journal-Bearing Faults: Making Use of Motor Current Signature Analysis for Induction Motors," IEEE Industry Applications Magazine, vol. 23, no. 4, pp. 12-21, 2017.

[11] L. B. Visnadi and H. F. de Castro, "Influence of bearing clearance and oil temperature uncertainties on the stability threshold of cylindrical journal bearings," Mechanism and Machine Theory, vol. 134, pp. 57-73, 2019.

[12] K. K. Yadav et al., "Studies and Analysis of Effect of Foreign Particles on the Parts of Steam Turbine," International Journal of Applied Engineering Research, vol. 13, no. 6, pp. 386-395, 2018.

[13] R. Ranjan, S. K. Ghosh, and M. Kumar, "Fault diagnosis of journal bearing in a hydropower plant using wear debris, vibration and temperature analysis: A case study," Journal of Process Mechanical Engineering, vol. 234, no. 3, pp. 235-242, 2020.

[14] Y. Elyassami, K. Benjelloun, and M. E. Aroussi, "Sleeve Bearing Fault Diagnosis and Classification," WSEAS Transactions On Signal Processing, vol. 12, pp. 2224-3488, 2016.

[15] H. Oh, J. H. Jung, B. C. Jeon, and B. D. Youn, "Scalable and Unsupervised Feature Engineering Using Vibration-Imaging and Deep Learning for Rotor System Diagnosis," IEEE Transactions on Industrial Electronics, vol. 65, no. 4, pp. 3539-3549, 2017.

[16] T. Narendiranath Babu, H. S. Himamshu, K. N. Prabin, P. D. Rama, and C. Nishant, "Journal Bearing Fault Detection Based on Daubechies Wavelet," Archives of Acoustics, vol. 42, no. 3, pp. 401- 414, 2017.

[17] T. Narendiranath Babu, T. Manvel Raj, and T. Lakshmanan, "Application of Butterworth filter for fault diagnosis on journal bearing," Journal of VibroEngineering, vol. 16, no. 3, pp. 1602-1617, 2014.

[18] T. Narendiranath Babu, A. Aravind, A. Rakesh, M. Jahzan, D. Rama Prabha, and M. R. Viswanathan, "Automatic Fault Classification for Journal Bearings Using ANN and DNN," Archives of Acoustics, vol. 43, no. 4, pp. 727-738, 2018.

[19] B. C. Jeon, "Statistical Approach to Diagnostic Rules for Various Malfunctions of Journal Bearing System Using Fisher Discriminant Analysis," in European Conference of the prognostics and health management society, 2014.

[20] J. H. J. Byung Chul Jeon, Byeng Dong Youn, Yeon-Whan Kim, and Yong-Chae Bae, "Datum Unit Optimization for Robustness of a Journal Bearing Diagnosis System," International Journal of Precision Engineering and Manufacturing, vol. 16, no. 11, pp. 2411-2425, 2015.

[21] J. Joon Ha, J. Byung Chul, Y. D. Byeng, K. Myungyon, K. Donghwan, and K. Yeonwhan, "Omnidirectional regeneration (ODR) of proximity sensor signals for robust diagnosis of journal bearing systems," Mechanical Systems and Signal Processing, vol. 90, pp. 189-207, 2017.

[22] H. Oh, B. C. Jeon, J. H. Jung, and B. D. Youn, "Smart diagnosis of journal bearing rotor systems: Unsupervised feature extraction scheme by deep learning," in Annual Conference of the Prognostics and Health Management Society, 2016.

[23] B. C. Jeon, J. H. Jung, M. Kim, K. H. Sun, and B. D. Youn, "Optimal vibration image size determination for convolutional neural network 
based fluid-film rotor-bearing system diagnosis," Journal of Mechanical Science and Technology, vol. 34, no. 4, pp. 1467-1474, 2020.

[24] N. Mokhtari and C. Gühmann, "Classification of journal bearing friction states based on acoustic emission signals," tm-Technisches Messen, vol. 85, no. 6, pp. 434-442, 2018.

[25] N. Mokhtari, J. G. Pelham, S. Nowoisky, J.-L. Bote-Garcia, and C. Gühmann, "Friction and Wear Monitoring Methods for Journal Bearings of Geared Turbofans Based on Acoustic Emission Signals and Machine Learning," Lubricants, vol. 8, no. 3, p. 27, 2020.

[26] J.-L. Bote-Garcia, N. Mokhtari, and C. Gühmann, "Wear monitoring of journal bearings with acoustic emission under different operating conditions," in PHM Society European Conference, 2020.

[27] S. Guo, T. Yang, W. Gao, and C. Zhang, "A Novel Fault Diagnosis Method for Rotating Machinery Based on a Convolutional Neural Network," Sensors, vol. 18, no. 5, pp. 1429-1444, 2018.

[28] S. Hosseini, M. Ahmadi Najafabadi, and M. Akhlaghi, "Classification of acoustic emission signals generated from journal bearing at different lubrication conditions based on wavelet analysis in combination with artificial neural network and genetic algorithm," Tribology International, vol. 95, pp. 426- 434, 2016.

[29] A. Moosavian, H. Ahmadi, and A. Tabatabaeefar "Fault Diagnosis of main engine journal bearing based on vibration analysis using Fisher linear discriminant, K-nearest neighbor and support vector machine," Journal of Vibroengineering vol. 14, no. 2, pp. 894-906, 2012.

[30] A. Moosavian, H. Ahmadi, A. Tabatabaeefar, and B. Sakhaei, "An Appropriate Procedure for Detection of Journal-Bearing Fault Using Power Spectral Density, K-Nearest Neighbor and Support Vector Machine," International Journal on Smart Sensing and Intelligent Systems, vol. 5, no. 3, pp. 685-700, 2012.

[31] A. Moosavian, "Comparison of two classifiers; K-nearest neighbor and artificial neural network, for fault diagnosis on a main engine journalbearing," Shock and Vibration, vol. 20, no. 2, pp. 263-272, 2013.

[32] D. Stuani Alves et al., "Uncertainty quantification in deep convolutional neural network diagnostics of journal bearingss with ovalization fault," Mechanism and Machine Theory, vol. 149, 2020.

[33] O. Gecgel et al., "Simulation-Driven Deep Learning Approach for Wear Diagnostics in Hydrodynamic Journal Bearings," Journal of Tribology, vol. 143 , no. 8, p. 9, 2020.

[34] S. M. DeCamilo, A. Dadouche, and M. Fillon, "Journal Bearings in Power Generation," in Encyclopedia of Tribology: Springer, 2013.

[35] S. Chatterton, P. Vinh Dang, P. Pennacchi, A. De Luca, and F. Flumian, "Experimental evidence of a two-axial groove hydrodynamic journal bearing under severe operation conditions," Tribology International, vol. 109, pp. 416-427, 2017.

[36] A. Muszynska, "Vibrational Diagnostics of Rotating Machinery Malfunctions," International Journal of Rotating Machinery, vol. 1, no. 34, pp. 237-266, 1995.

[37] A. Bilošová and J. Biloš, Vibrations Diagnostics. Ostrava: VSB Technical University of Ostrava, 2012.

[38] J. Pino Gómez, F. E. Hernández Montero, and J. C. Gómez Mancilla, "Variable Selection for Journal Bearing Faults Diagnostic Through Logical Combinatorial Pattern Recognition," in Lecture Notes in Computer Science: Springer, 2018.
[39] J. Ruiz-Shulcloper, "Pattern Recognition with Mixed and Incomplete Data," Pattern Recognition and Image Analysis, vol. 18, no. 4, pp. 563$576,2008$.

[40] V. Rodríguez-Diez, J. F. Martínez-Trinidad, M. S. Lazo-Cortés, and J. A. Carrasco-Ochoa, "The Impact of Basic Matrix Dimension on the Performance of Algorithms for Computing Typical Testors," in Lecture Notes in Computer Science: Springer, 2018.

[41] V. Rodríguez-Diez, J. F. Martínez-Trinidad, M. S. Lazo-Cortés, and J. A. Carrasco-Ochoa, "A new algorithm for reduct computation based on gap elimination and attribute contribution," Information Sciences, vol. 435, pp. 111-123, 2018.

[42] T. Cover and P. Hart, "Nearest neighbor pattern classification," IEEE Transactions on Information Theory, vol. 13, no. 1, pp. 21-27, 1967.

[43] Y. Villuendas-Rey, C. F. Rey-Benguría, Á. Ferreira-Santiago, O. Camacho-Nieto, and C. Yáñez-Márquez, "The Naïve Associative Classifier (NAC): A novel, simple, transparent, and accurate classification model evaluated on financial data," Neurocomputing vol. 265, pp. 105-115, 2017.

[44] Y. Villuendas-Rey, M.-D. Alanis-Tamez, C.-F. Rey Benguría, C. YáñezMárquez, and O. Camacho-Nieto, "Medical Diagnosis of Chronic Diseases Based on a Novel Computational Intelligence Algorithm," Journal of Universal Computer Science, vol. 24, no. 6, pp. 775-796, 2018.

[45] D. R. Wilson and T. R. Martinez, "Improved heterogeneous distance functions," Journal of Artificial Intelligence Research, vol. 6, pp. 1-34, 1997.

[46] J. A. Hernández-Castaño, O. Camacho-Nieto, Y. Villuendas-Rey, and C. Yáñez Márquez, "Experimental Platform for Intelligent Computing (EPIC)," Computación y Sistemas, vol. 22, no. 1, pp. 245-253, 2018.

[47] M. Hall, E. Frank, G. Holmes, B. Pfahringer, P. Reutemann, and I. H. Witten, "The WEKA Data Mining Software: An Update," ACM SIGKDD explorations newsletter, vol. 11, no. 1, pp. 10-18, 2009.

[48] J. Alcalá-Fdez et al., "KEEL Data-Mining Software Tool: Data Set Repository, Integration of Algorithms and Experimental Analysis Framework," Journal of Multiple-Valued Logic \& Soft Computing, vol. 17, pp. 255-287, 2011.P. Refaeilzadeh, L. Tang, and H. Liu, "CrossValidation," in Encyclopedia of Database Systems: Springer, 2009.

[49] M. Sokolova, N. Japkowicz, and S. Szpakowicz, "Beyond Accuracy, FScore and ROC: A Family of Discriminant Measures for Performance Evaluation," in Lecture Notes in Computer Science: Springer, 2006.

[50] M. Kubat, R. Holte, and S. Matwin, "Learning when negative examples abound," in European Conference on Machine Learning, 1997.

[51] M. Sokolova and G. Lapalme, "A systematic analysis of performance measures for classification tasks," Information Processing \& Management, vol. 45, no. 4, pp. 427-437, 2009.

[52] M. Kubat and S. Matwin, "Addressing the curse of imbalanced training sets: one-sided selection," in International Conference on Machine Learning, 1997.

[53] D. Ballabio, F. Grisoni, and R. Todeschini, "Multivariate comparison of classification performance measures," Chemometrics and Intelligent Laboratory Systems, vol. 174, pp. 33-44, 2018. 